\title{
Controlling the quality of nanocrystalline silicon made by hot-wire chemical vapor deposition by using a reverse $\mathrm{H}_{2}$ profiling technique
}

\author{
H. Li, R.H. Franken, R.L. Stolk, C.H.M. van der Werf, J.K. Rath, R.E.I. Schropp* \\ Faculty of Science, Utrecht University, SID - Physics of Devices, P.O. Box 80 000, 3508 TA Utrecht, The Netherlands
}

Available online 29 January 2008

\begin{abstract}
Hydrogen profiling, i.e., decreasing the $\mathrm{H}_{2}$ dilution during deposition, is a well-known technique to maintain a proper crystalline ratio of the nanocrystalline (nc-Si:H) absorber layers of plasma-enhanced chemical vapor-deposited (PECVD) thin film solar cells. With this technique a large increase in the energy conversion efficiency is obtained. Compared to PECVD, the unique characteristics of hot-wire CVD (HWCVD), such as the catalytic reactions, the absence of ion bombardment, the substrate heating by the filaments and filament aging effects, necessitate a different strategy for material and device optimization. We report in this paper the results of using a reverse $\mathrm{H}_{2}$ profiling technique, i.e., increasing the $\mathrm{H}_{2}$ dilution of silane instead of decreasing it, to improve the quality of HWCVD intrinsic nc-Si:H and the performance of this material in single junction n-i-p cells. Thus far, the efficiency of nc-Si:H n-i-p cells made on a stainless steel substrate with an $\mathrm{Ag} / \mathrm{ZnO}$ textured back reflector has been improved to 8.5\%, and the efficiency of triple junction solar cells with a structure of proto-Si:H(HWCVD) top cell/proto-SiGe:H (PECVD) middle cell/nc-Si:H (HWCVD, with reverse $\mathrm{H}_{2}$ profiling) bottom cell has reached $10.9 \%$. These efficiency values show the viability of n-i-p cells comprising HWCVD nanocrystalline i-layers.
\end{abstract}

(C) 2007 Elsevier B.V. All rights reserved.

PACS: $68.55 ; 73.50 . \mathrm{Pz} ; 73.63 . \mathrm{Bd} ; 81.15 .-\mathrm{Z}$

Keywords: Silicon; Solar cells; Nanocrystals; Photovoltaics; Chemical vapor deposition; Microcrystallinity

\section{Introduction}

Hydrogenated nanocrystalline silicon (nc-Si:H) has been intensively studied for photovoltaic applications because of its better stability against prolonged illumination than amorphous silicon (a-Si:H) and amorphous silicon germanium (a-SiGe:H) [1-3]. The conventional techniques of producing such material are based on plasma enhanced chemical vapor deposition (PECVD), and much research is dedicated to optimizing the plasma conditions for highrate deposition. In this paper, we present materials and devices deposited by hot-wire chemical vapor deposition (HWCVD) [4], because of its unique properties such as

\footnotetext{
* Corresponding author. Tel.: +31 30 2533170; fax: +31 302543165 .

E-mail addresses: h.li@phys.uu.nl (H. Li), R.E.I.Schropp@phys.uu.nl (R.E.I. Schropp).
}

the absence of ion bombardment, the lack of any dependence of the gas phase reactions on the electromagnetic properties of the substrate, the low dust formation in a broad parameter space, the straightforward possibilities for scaling up, and the potential of producing device quality materials at a high deposition rate [5].

In a conventional $\mathrm{p}-\mathrm{i}-\mathrm{n}$ configured solar cell structure, intrinsic nc-Si:H is used as the active layer (or one of the active layers) where electron and holes are generated due to light absorption. To effectively collect these photogenerated carriers in an i-layer that is thick enough to absorb most of the incident light, a minimal electrical defect density is required. It has been shown by many groups that the best solar cells contain nc-Si:H i-layers that are made in the regime near the transition between amorphous and microcrystalline silicon $(\mu \mathrm{c}-\mathrm{Si}: \mathrm{H})$, and at a relatively low substrate temperature around $200{ }^{\circ} \mathrm{C}$ where the opto-elec- 
tronic properties are optimal. From the device performance point of view, a shift of the deposition parameters to either side of the transition regime will eventually lead to a decrease in carrier generation (and thus a reduced short circuit current density $J_{\mathrm{sc}}$ ) if the shift is to the a-Si:H side, or a deterioration in effective carrier collection (resulting in low open circuit voltage $V_{\text {oc }}$ and fill factor $F F$ ) if the shift is too far into the $\mu \mathrm{c}-\mathrm{Si}: \mathrm{H}$ side [6]. It is therefore important to carefully control the deposition parameters in order to keep the material structure optimal. This contribution presents the result of our study in this respect in order to develop high quality nc-Si:H single junction n-i-p solar cells with hot-wire CVD deposited i-layers.

\section{Experimental details}

The solar cells discussed in this article have a structure of substrate (Asahi U-type glass or stainless steel/back reflector (BR))/n-type nc-Si:H/nc-Si:H i-layer/p-type nc$\mathrm{Si}: \mathrm{H} / \mathrm{ITO} / \mathrm{Au}$ grid. The silicon layers were deposited in a multi-chamber UHV cluster system called PASTA - intrinsic layers by HWCVD and doped layers with conventional RF-PECVD at $13.56 \mathrm{MHz}$. HWCVD was performed using two straight tantalum wires $(0.5 \mathrm{~mm}$ in diameter $)$ that were about $4 \mathrm{~cm}$ from the substrate. A constant current of $10.5 \mathrm{~A}$ was used, yielding a wire temperature of around $1850^{\circ} \mathrm{C}$ in vacuum (measured with a pyrometer). No extra substrate heating was applied, which resulted in a substrate temperature of around $270{ }^{\circ} \mathrm{C}$ when the system is in an equilibrium condition. Raman spectroscopy at a laser wavelength of $514.5 \mathrm{~nm}$ was employed to estimate the crystalline ratio of absorber layers as well as of complete solar cells. A structural transition between $\mu \mathrm{c}-\mathrm{Si}: \mathrm{H}$ and a-Si:H has been observed at a gas flow ratio of $R_{\mathrm{H}}=\mathrm{H}_{2} /$ $\left(\mathrm{SiH}_{4}+\mathrm{H}_{2}\right)=0.95$ [6]. Silicon n-i-p stacks deposited on accompanying Corning 1737 glass substrates were investigated with cross-sectional transmission electron microscopy (XTEM). The same samples were also used for thickness determination by fitting the optical reflection/ transmission spectra or by using a surface step profiler (Dektak). The current density-voltage $(J-V)$ characteristics of the solar cells were measured at $25^{\circ} \mathrm{C}$ under AM1.5 $100 \mathrm{~mW} / \mathrm{cm}^{2}$ white light generated by a dual beam solar simulator (WACOM). A $0.3 \mathrm{~mm}$ thick SS mask used for depositing the ITO top contact was also used during the measurement to have a precise definition of the cell area.

\section{Results and discussion}

\subsection{Structural variation of $n c-S i$ :H materials grown in the phase transition regime}

To obtain more insight into the structural evolution of nc-Si:H i-layers along the growth direction, solar cells with various i-layer thickness have been made. Besides the ilayer deposition time, all other parameters were kept constant within each series. All measurements were performed near the centre of the substrate. Raman spectroscopy was used to estimate the i-layer crystallinity for both the samples deposited on SS and the samples deposited on the accompanying Corning 1737 glass, with the excitation laser incident from the $\mathrm{p}$-side of the cell at a location where no ITO contact is present. Due to the very thin p-layer thickness used ( $20 \mathrm{~nm})$, the Raman signal is dominated by the nc-Si:H i-layer. The influence from the p-layer on thus estimated structural variation among the samples has been checked to be negligible. Crystalline ratio $X_{\mathrm{c}}$ is defined as the integrated area ratio between the crystalline $\mathrm{Si}$ TO peak and the a-Si:H TO peak $\left(\mathrm{I}_{520}+\mathrm{I}_{510}\right) /\left(\mathrm{I}_{520}+\mathrm{I}_{510}+\mathrm{I}_{480}\right)$. Fig. 1 shows the Raman crystalline ratio $X_{\mathrm{c}}$ dependence on the i-layer thickness. Two hydrogen dilution ratios $R_{\mathrm{H}}$ were used for the samples shown in Fig. 1. The squares
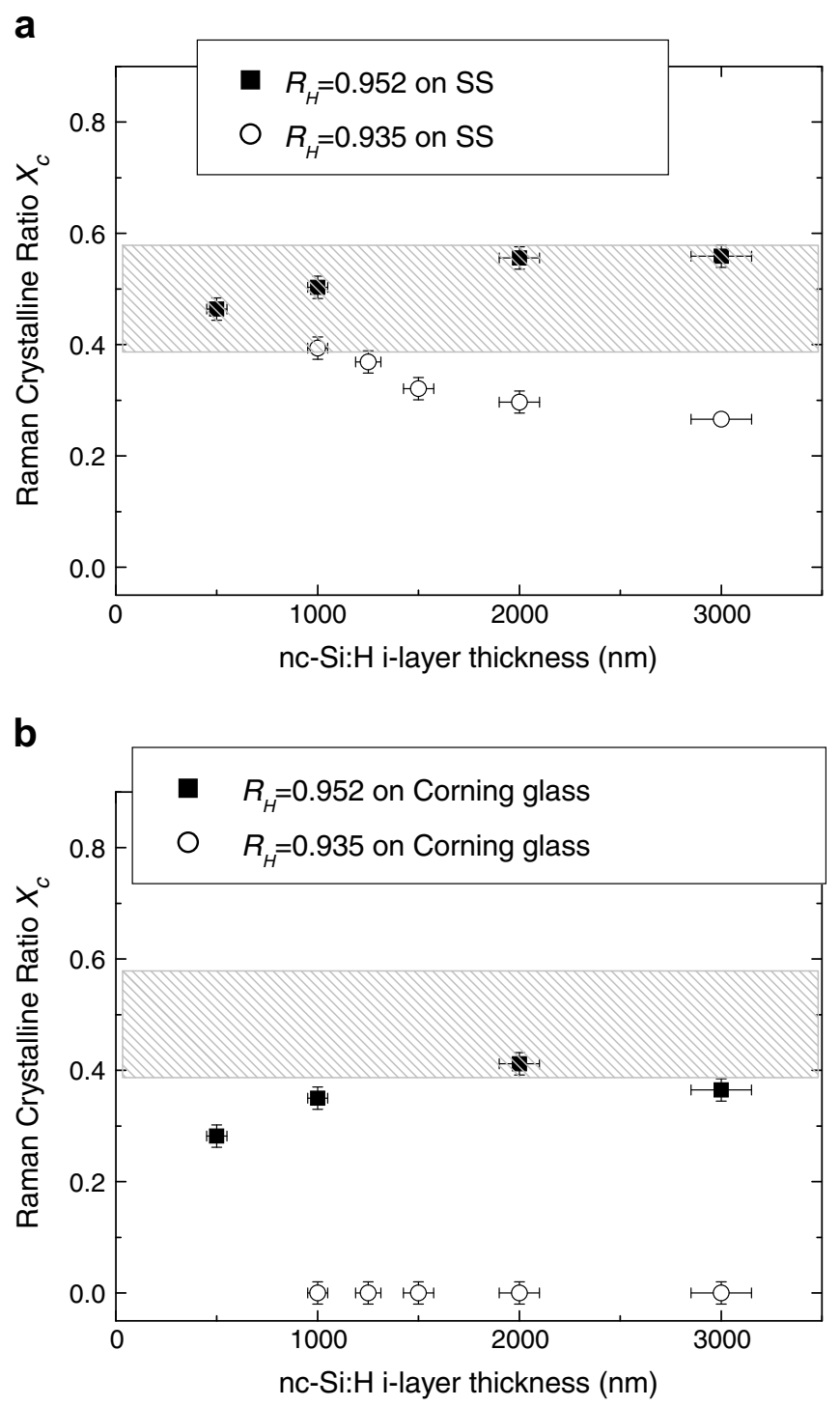

Fig. 1. i-layer thickness dependence of Raman crystalline ratio $X_{\mathrm{c}}$ for n-ip cells deposited on stainless steel foil (a) and Corning 1737 glass sheet (b). Values shown in the legend are the $\mathrm{H}_{2}$ dilution ratio $R_{\mathrm{H}}$. The grey area represents the reported $X_{\mathrm{c}}$ values of the nc-Si:H solar cells showing the best performance. 
are for $R_{\mathrm{H}}=0.952$, the circles for $R_{\mathrm{H}}=0.935$. Fig. 1(a) shows the samples deposited on $\mathrm{SS} / \mathrm{Ag} / \mathrm{ZnO}$ substrates, Fig. 1(b) those on the accompanying Corning 1737 glass. Clearly, the materials develop very differently, depending on the type of substrate. This is a well-known substrate dependent effect, which in this case can be attributed to the differences in substrate temperature, in substrate surface roughness, and in the structure of the PECVD deposited n-layer.

A more interesting observation in Fig. 1 is that, depending on the value of $R_{\mathrm{H}}$ and substrate type, the material crystallinity shows a different trend with increasing i-layer thickness. For samples deposited on $\mathrm{SS}$ with a $R_{\mathrm{H}}$ value of $0.952, X_{\mathrm{c}}$ shows a slight increase with increasing i-layer thickness from $\sim 0.5 \mu \mathrm{m}$ to $\sim 2 \mu \mathrm{m}$, and seems to saturate after $2 \mu \mathrm{m}$ of thickness. For the $R_{\mathrm{H}}=0.935$ series, $X_{\mathrm{c}}$ starts at a value around 0.4 for the cell with a $1-\mu \mathrm{m}$ thick i-layer, and continuously decreases with increasing the thickness. This is somewhat unexpected, since it is widely observed for silicon materials prepared with PECVD that the crystallinity of $\mu \mathrm{c}-\mathrm{Si}: \mathrm{H}$ increases with layer thickness, as has been summarized by Collins et al. in Ref. [7], and is represented in Fig. 2 (top part). The slight increase in crystallinity of the $R_{\mathrm{H}}=0.952$ series in the thickness range of 0.5 $2 \mu \mathrm{m}$ could be explained by the existence of an incubation phase where a more amorphous region exists near the substrate. Although the incubation phase may also exist for the layers deposited with a $R_{\mathrm{H}}$ ratio of 0.935 , the continuous decrease of $X_{\mathrm{c}}$ with increasing i-layer thickness for that series is unexpected.

To check if the samples indeed have such breakdown in crystalline structure, cross sectional TEM specimen were prepared using the samples deposited on Corning glass. Fig. 2 (lower part) shows TEM pictures for samples made at a few different $R_{\mathrm{H}}$ (from left to right, $R_{\mathrm{H}}=0.926,0.935$, 0.971 , respectively - low dilution to high dilution) that best illustrate the typical evolution of our HWCVD films. The micrograph of the sample deposited at the highest $R_{\mathrm{H}}$ (0.971) shows a highly crystalline structure over the whole i-layer, whereas for $R_{\mathrm{H}}=0.926$ the i-layer is completely amorphous. For the sample deposited with $R_{\mathrm{H}}=0.935$ a clear though not sharp transition from crystalline to amorphous structure is observed. The thickness where this transition takes place is between 500 and $700 \mathrm{~nm}$ for a sample made on Corning glass. The films that are grown on the SS substrate likely have a thicker crystalline part than those grown on the Corning glass substrate. The development of amorphous phase towards the top of the i-layer can therefore be observed by the Raman scattering measurements of the films grown on SS when increasing the layer thickness (Fig. 1(a)). On the other hand, since the penetration depth of the laser light used for the Raman scattering measurement is limited to a few hundred nanometres in silicon, the Raman measurement from the p-side of the sam-

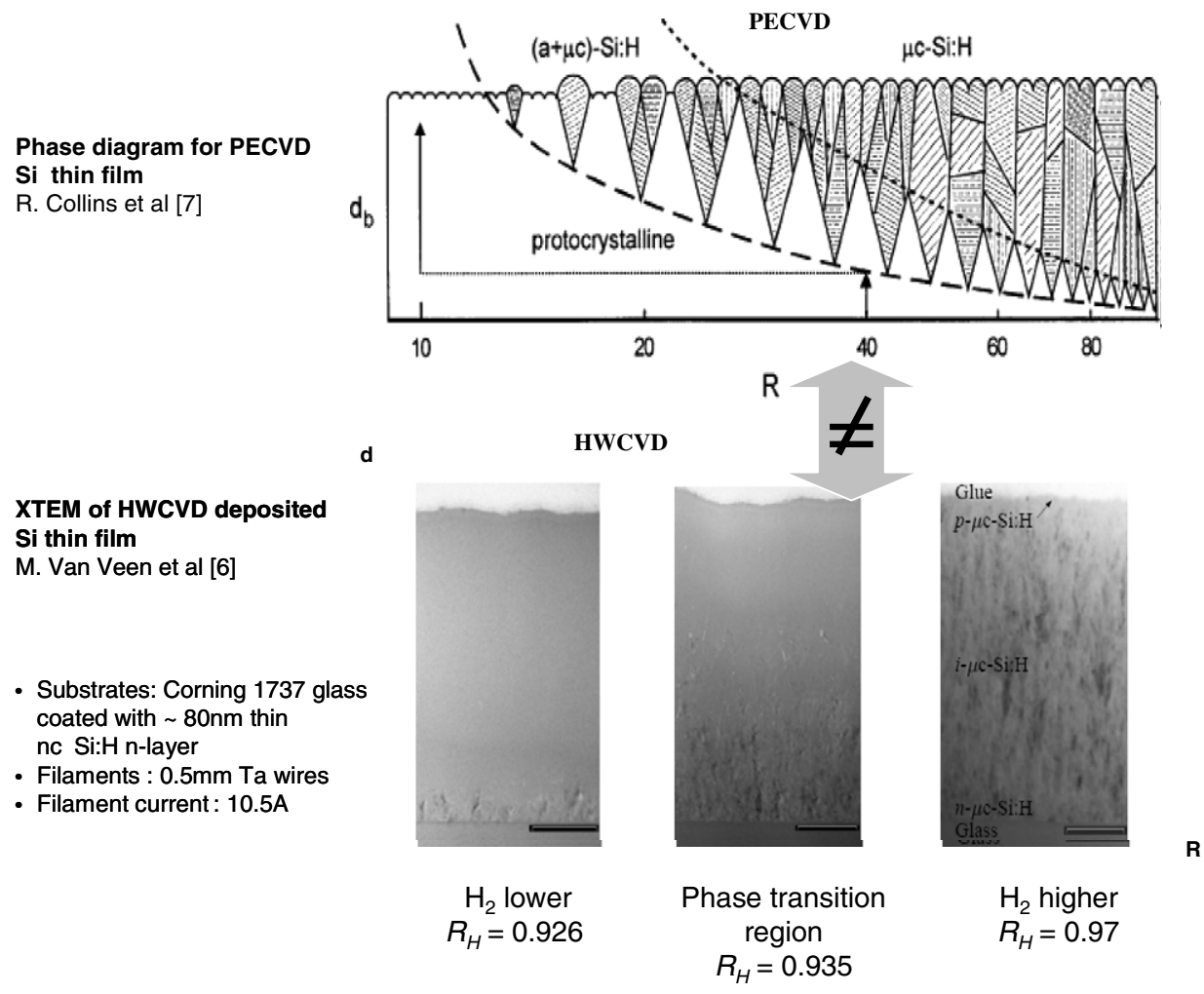

Fig. 2. The structure of nc-Si:H film deposed by hot-wire CVD (lower part) shows a different trend with increasing $\mathrm{H}_{2}$ dilution compared to that by conventional PECVD (upper part, reprinted from [7], Copyright 2002, with permission from Elsevier). The scale bars shown in the micrographs are $200 \mathrm{~nm}$. 
ple can only give information on the upper part of the n-i-p stacks. Therefore, the $X_{\mathrm{c}}$ values estimated from the Raman scattering patterns of the $R_{\mathrm{H}}=0.935$ samples deposited on Corning glass are all close to zero (Fig. 1(b)), meaning that the upper part of the n-i-p stack is purely amorphous, like that shown in Fig. 2 (lower part).

\subsection{Discussion}

The reverse development of nc-Si:H may have various causes. One of the possible origins might be the special features of the hot-wire CVD technique. Unlike the conventional plasma enhanced chemical vapor deposition (PECVD), source gases $\left(\mathrm{SiH}_{4}\right.$ and $\left.\mathrm{H}_{2}\right)$ are decomposed on the surface of a hot-filament by (thermo-) catalytic reactions, instead of via collisions of high-energy electrons with molecules in the gas phase. An important aspect is the absence of energetic ions reaching the surface of the growing film. The ions in PECVD are believed to contribute to the surface reactions in PECVD, in addition to hydrogen and silicon containing radicals. In the case of HWCVD, not only the ions are almost completely absent, but also the variety of radicals reaching the surface is much smaller. While moving toward the substrate, silicon atoms further react with gas molecules, forming silicon hydrides such as $\mathrm{SiH}, \mathrm{SiH}_{2}, \mathrm{SiH}_{3}$ or $\mathrm{Si}_{2} \mathrm{H}_{4}[8,9]$. The relative flux of each radical as well as the film surface temperature, determines the growth of silicon layers.

The driving force for hot-wire chemical vapor deposition is the reactions taking place on the hot-wire surface. The most important reaction taking place on the filament surface is the silane dissociation reaction, namely $\mathrm{SiH}_{4}=$ $\mathrm{Si}+4 \mathrm{H}$. While this reaction is mainly catalytic, an increasing fraction of the reactions may be due to pyrolytic dissociation at the filament when it is ageing [10-12].

For a fresh filament at a high enough filament temperature, all silane molecules are decomposed within a few collisions with the hot-wire surface, resulting in a significant amount of atomic $\mathrm{Si}$ and $\mathrm{H}$ evaporating to the gas phase [10]. At a moderate filament temperature, most of the $\mathrm{SiH}_{4}$ molecules are also dissociated upon collision with the filament surface, but the evaporation of silicon atoms becomes slower, as insufficient energy is available for silicon to immediately desorb from the filament surface. A silicon layer therefore accumulates on the filament surface. The accumulated silicon atoms can react with or diffuse into the metal wires, forming metal silicon alloy or metal silicide. At an even lower temperature or at a very high silane partial pressure, depending on the type of filament, liquid silicon may appear on the filament surface, which further reduces the filament's catalytic ability $[10,13]$.

Such a filament condition dependence of hot-wire CVD makes it reasonable for us to assume that the filament condition might have been changing during the film deposition. A good measure of the filament condition is the filament resistance. It has been reported that the resistance of the filament (tantalum in this case) increases after each deposition, for every filament used [6,14]. This is attributed mainly to silicidation of the filament during the catalytic reactions. This silicidation must have a dependence on silane partial pressure, which is determined by the source gas ratio $R_{\mathrm{H}}$ if the chamber pressure is kept unchanged during deposition. This led us to monitor the filament electrical properties during film deposition, which has resulted in an improved understanding of the reactions taking place at the filament surface. It is expected that better control of the filament conditions can be obtained as a result of this research.

Besides the possible reasons mentioned above, a strong influence of the nc-Si:H n-layer on the initial growth of intrinsic nc-Si:H has to be considered. As was mentioned earlier, the nc-Si:H n-layer promotes the growth of ncSi:H i-layer so that the highly amorphous incubation phase that normally appears in the silicon layers near the substrate (shown in the upper part of Fig. 2) is not observed in the XTEM picture of this set of samples (shown in the lower part of Fig. 2). It is reasonable to assume that the influence of this 'substrate memory effect' decreases and eventually vanishes as increasing the i-layer thickness, which causes the breakdown of nanocrystalline silicon growth due to the possibly unfavourable condition at $R_{H}=0.948$ for nc-Si:H growth.

The above mentioned mechanisms may act side by side on the growth of nc-Si:H made with hot-wire CVD in the transition regime. A clear understanding of to what extent each mechanism contributes to the material growth needs obviously more detailed study. To our knowledge, such a nanocrystalline breakdown has not been reported for solar cells with nc-Si:H absorber layers made with plasma enhanced CVD (either RF or VHF). Further study on this phenomenon is therefore interesting for the comparison of these two techniques on their various applications.

\subsection{Solar cell with reverse $\mathrm{H}_{2}$ profiling}

Based on the above observations, $n-i-p$ cells with a nc$\mathrm{Si}: \mathrm{H}$ i-layer were made near the phase transition regime while avoiding structural changes during deposition. A way to control the structural evolution in the nc-Si:H silicon cells is the use of a hydrogen profiling technique. In PECVD, $R_{\mathrm{H}}$ is decreased during i-layer deposition [15] to suppress the development of the deposited nc-Si:H i-layers toward higher crystallinity. Using this technique, a general increase in all the cell parameters was observed, thus improving the solar cell efficiency considerably $[15,16]$. In our case, since the i-layer structure develops in the opposite direction, we used a reverse $\mathrm{H}_{2}$ profiling scheme, namely an increase of the $R_{\mathrm{H}}$ while depositing the i-layer, instead of decreasing it. The preliminary result is encouraging [17]. Fig. 3 shows the AM1.5 $J-V$ characteristics of several $\mu$ c$\mathrm{Si}: \mathrm{H}$ n-i-p cells; the cell output parameters are listed in Table 1. 'Cold start' means that the i-layer deposition was started before the chamber reached a thermal equilibrium condition. All cell parameters have improved drasti- 


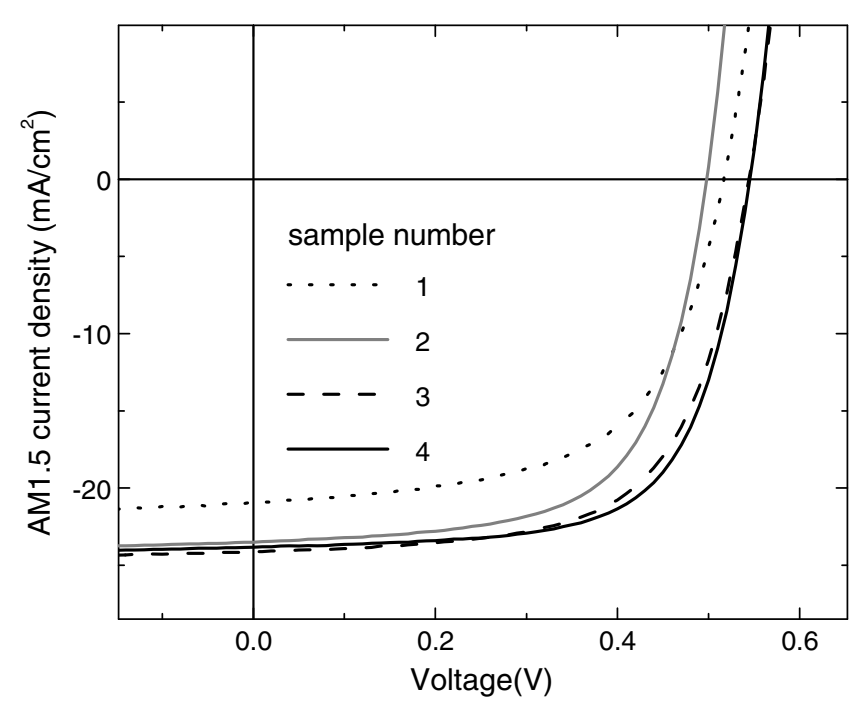

Fig. 3. AM1.5 $J-V$ characteristics of nc-Si:H n-i-p cells on $\mathrm{SS}$ with a Ag/ ZnO TBR. (1) constant $\mathrm{H}_{2}$ dilution at $R_{\mathrm{H}}=0.952$; (2) constant $\mathrm{H}_{2}$ dilution at $R_{\mathrm{H}}=0.948$; (3) two step profiling, 1st half at $R_{\mathrm{H}}=0.948,2$ nd half at $R_{\mathrm{H}}=0.952$; (4) with the same $\mathrm{H}_{2}$ profile as (3), but with a cold start. The i-layer deposition rate is around $2 \AA / \mathrm{s}$, the thickness $\sim 2 \mu \mathrm{m}$.

Table 1

AM1.5 output parameters of the nc-Si:H n-i-p cells shown in Fig. 3

\begin{tabular}{llllll}
\hline $\begin{array}{l}\text { Sample } \\
\text { no. }\end{array}$ & Remarks & $\begin{array}{l}\text { Efficiency } \\
(\%)\end{array}$ & $\begin{array}{l}J_{\mathrm{sc}} \\
\left(\mathrm{mA} / \mathrm{cm}^{2}\right)\end{array}$ & $\begin{array}{l}V_{\mathrm{oc}} \\
(\mathrm{V})\end{array}$ & $F F$ \\
\hline 1 & $\begin{array}{l}\text { Without } \mathrm{H}_{2} \text { profiling } \\
\left(R_{\mathrm{H}}=0.952\right)\end{array}$ & 7.5 & 23.5 & 0.498 & 0.641 \\
2 & $\begin{array}{l}\text { Without } \mathrm{H}_{2} \text { profiling } \\
\left(R_{\mathrm{H}}=0.948\right)\end{array}$ & 6.5 & 21.2 & 0.517 & 0.594 \\
3 & $\begin{array}{l}\text { With two step } \mathrm{H}_{2} \\
\text { profiling }\left(R_{\mathrm{H} 1}=0.948,\right. \\
\left.R_{\mathrm{H} 2}=0.952\right)\end{array}$ & 8.4 & 24.2 & 0.544 & 0.636 \\
& $\begin{array}{l}\text { Two step } \mathrm{H}_{2} \text { profiling }+ \\
\text { cold start }\end{array}$ & 8.5 & 23.4 & 0.545 & 0.668 \\
\hline
\end{tabular}

cally by applying this 'reverse profiling'. The cell efficiency has reached $8.5 \%$, which is the highest value reported so far for a nc-Si:H n-i-p cell with a HWCVD i-layer.

\section{Conclusion}

In this article, we presented our results on using a reverse $\mathrm{H}_{2}$ profiling technique to improve the i-layer quality in single junction nc-Si:H thin film solar cells with an ilayer made by hot-wire CVD. This design is based on our study of the structural evolution of HWCVD deposited
nc-Si:H in the phase transition regime between a-Si:H and $\mu \mathrm{c}-\mathrm{Si}: \mathrm{H}$. Cells with an i-layer grown with this technique showed a record efficiency of $8.5 \%$ for single junction n-i-p cells with a HWCVD $\mu \mathrm{c}-\mathrm{Si}: \mathrm{H}$ i-layer. This new method allows the development of $\mu \mathrm{c}-\mathrm{Si}: \mathrm{H}$ cells with higher efficiency at higher deposition rate.

\section{Acknowledgements}

The research is financially supported by the Netherlands Agency for Energy and the Environment (SenterNovem) of the Ministry of Economic Affairs.

\section{References}

[1] J. Meier, R. Flückiger, H. Keppner, A. Shah, Appl. Phys. Lett. 65 (1994) 860.

[2] T. Repmann, B. Sehrbrock, C. Zahren, H. Siekmann, J. Müller, B. Rech, W. Psyk, R. Geyer, P. Lechner, in: Proceedings of 3rd World Conference on Photovoltaic Energy Conversion, Osaka, May 2003, 5O.A6.03.

[3] M. Yoshimi, T. Sasaki, T. Sawada, T. Suezaki, T. Meguro, T. Matsuda, K. Santo, K. Wadano, M. Ichikawa, A. Nakajima, K. Yamamoto, in: Proceedings of 3rd World Conference on Photovoltaic Energy Conversion, Osaka, May 2003, 5O.A6.01.

[4] R.E.I. Schropp, Thin Solid Film 451\&452 (2004) 455.

[5] H. Li, Single and multijunction silicon based thin film solar cells on flexible substrates with absorber layers made by hot-wire CVD, $\mathrm{PhD}$ thesis, Utrecht University, 2007.

[6] M. van Veen, Tandem solar cells deposited using hot-wire chemical vapor deposition, $\mathrm{PhD}$ thesis, Utrecht University, The Netherlands, 2003.

[7] R.W. Collins, A.S. Ferlauto, Curr. Opin. Sol. Stat. \& Mat. Sci. 6 (2002) 425.

[8] Alan Gallagher, Thin Solid Film 395 (2001) 25.

[9] Y. Nozaki, M. Kitazoe, K. Horii, H. Umemoto, A. Masuda, H. Matsumura, Thin Solid Film 395 (2001) 47.

[10] R. Doyle, R. Robertson, G. Lin, M. He, A. Gallagher, J. Appl. Phys. 64 (1988) 3215.

[11] H.L. Duan, G.A. Zaharias, Stacey F. Bent, Mat. Res. Soc. Symp. Proc. 715 (2002) A15.5.7.

[12] J.K. Holt, Hot-wire chemical vapor deposition of silicon and silicon nitride for photovoltaics: experiments, simulations, and applications, $\mathrm{PhD}$ thesis, California Institute of Technology, 2003.

[13] H.L. Duan, G.A. Zaharias, Stacey F. Bent, Thin Solid Film 395 (2001) 36

[14] D. Grunsky, M. Kupich, B. Hofferberth, B. Schroeder, Thin Solid Film 501 (2006) 332.

[15] B. Yan, G. Yue, J. Yang, S. Guha, D.L. Williamson, D. Han, C. Jiang, Appl. Phys. Lett. 85 (2004) 1955.

[16] A. Gordijn, J.K. Rath, R.E.I. Schropp, Prog. Photovoltaics: Res. Appl. 14 (4) (2006) 305.

[17] H. Li, R.H. Franken, R.L. Stolk, C.H.M. van der Werf, J.K. Rath, R.E.I. Schropp, Thin Solid Film 516 (2008) 755. 